\title{
Supporting Information: Influence of Confinement on the Chain Conformation of Cyclic Poly(N-Isopropylacrylamide)
}

\author{
David Magerl ${ }^{\dagger}$, Martine Philipp ${ }^{\dagger}$, Ezzeldin Metwalli ${ }^{\dagger}$, Philipp Gutfreund ${ }^{\ddagger}$, Xing-Ping Qiu $^{\S}$, \\ Françoise M. Winnik ${ }^{\S, \#}$, and Peter Müller-Buschbaum*, ${ }^{\dagger}$
}

† Lehrstuhl für Funktionelle Materialien, Physik-Department, Technische Universität München, James-FranckStr. 1, 85748 Garching, Germany

‡ Institut Laue-Langevin, 71 avenue des Martyrs, 38000 Grenoble, France

$\S$ Faculty of Pharmacy and Department of Chemistry, Université de Montréal, CP 6128 Succursale Centre Ville, Montréal QC H3C 3J7, Canada

\# World Premier International (WPI) Research Center Initiative, International Center for Materials

Nanoarchitectonics (MANA) and National Institute for Materials Science (NIMS), 1-1 Namiki, Tsukuba 305-

0044, Japan

\section{Experimental}

Protonated cyclic poly(N-isopropylacrylamide) (PNIPAM) was prepared and characterized according to the method reported previously. ${ }^{1,2}$ The molecular weight of the linear precursor was determined by GPC (Mw $\left.=12.8 \mathrm{kDa}\right)$ and by end-group analysis using ${ }^{1} \mathrm{H}$ NMR and UV-visible spectroscopy $(\mathrm{Mn}=11.6 \mathrm{kDa})$, as described previously ${ }^{1}$. Identical procedures were used to prepare deuterated l-PNIPAM and c-PNIPAM by polymerization of hexadeuterated PNIPAM $\left[\left(\mathrm{CD}_{3}\right)_{2} \mathrm{CH}-\mathrm{NHCOCH}=\mathrm{CH}_{2}\right]$, itself obtained by reaction of acryloylchloride with hexadeuterated isopropylamine $\left[\left(\mathrm{CD}_{3}\right)_{2} \mathrm{CH}-\mathrm{NH}_{2}\right.$, Aldrich Chem. Corp.). The ${ }^{1} \mathrm{H}$ NMR spectra of l-PNIPAM(d6) and c-PNIPAM(d6) are presented in Figure S1. ${ }^{2}$ The molecular weight of the linear precursor l-PNIPAM(d6) was determined by GPC $(\mathrm{Mw}=12.7 \mathrm{kDa})$ and by end-group analysis using ${ }^{1} \mathrm{H}$ NMR and UV-visible spectroscopy (Mn = $\left.11.8 \mathrm{kDa}\right)$.

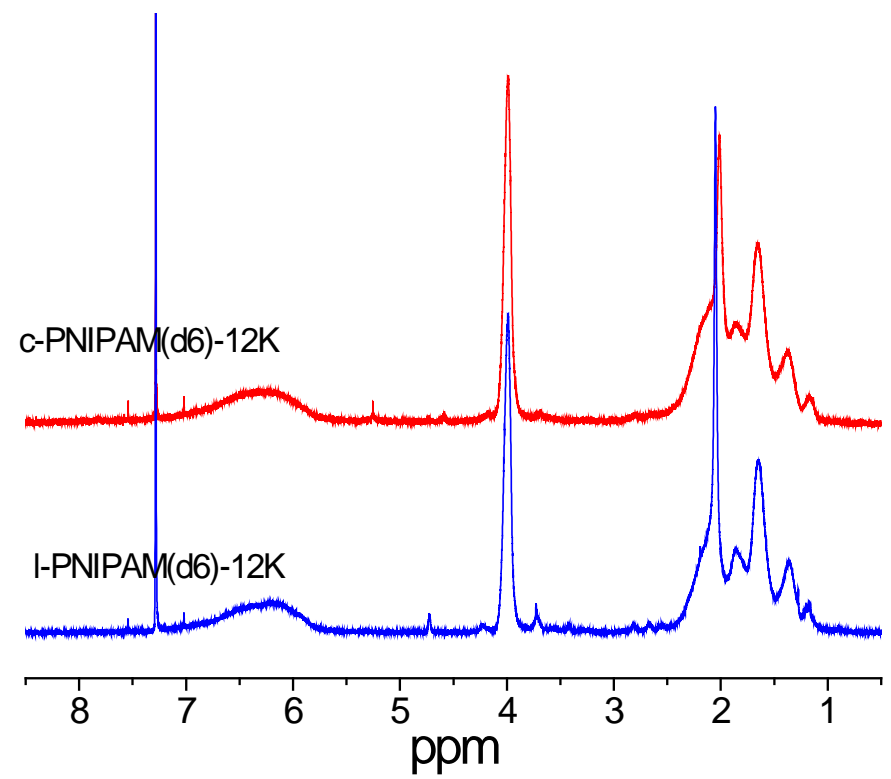

Figure S1. 1H NMR spectra of l-PNIPAM(d6)-12K and c-PNIPAM(d6)-12K in $\mathrm{CDCl}_{3}$. 
A dilute aqueous solution of the cyclic PNIPAM (5 mass\%) was prepared by dissolving the polymer in doubly distilled water, shaking it for one day and letting it equilibrate in the fridge for a week. The temperature of its demixing phase transition equals to $34^{\circ} \mathrm{C}$.

Films consisting of a blend of protonated and partially deuterated cyclic PNIPAM were prepared. In order to maximize the scattering contrast between the individual macromolecules, a ratio of 1:1 between the partially deuterated and the protonated cyclic PNIPAM was chosen. Segregation between protonated and partially deuterated chains was not expected and in accordance to our investigations did also not happen. The films of blends of protonated and deuterated PNIPAM were prepared by spin-coating the films out of chloroform solutions using a Süss MicroTec Delta6RC spin coater (2000 rpm, $30 \mathrm{~s}$ ). The thickness of the PNIPAM films can be accurately tuned by changing the solvent concentration of the polymer solution, as it typically depends in a linear manner on concentration. ${ }^{3}$ The chosen concentrations were $10.0,0.83$, and $0.16 \mathrm{~g} / \mathrm{L}$. The stability of ultrathin films is a most delicate issue as spinodal or binodal dewetting often occurs. ${ }^{4,5}$ Therefore, the polished silicon wafers underwent a special treatment, in order to create hydrophilic surfaces, which favor their wetting by PNIPAM. The treatment of the wafers included a cleaning step with a bath containing a mixture of $45 \mathrm{~mL}$ de-ionized water, $70 \mathrm{~mL}$ hydrogen peroxide (30\%) and $165 \mathrm{~mL}$ sulfuric acid (96\%). ${ }^{6}$ It is followed by a treatment with oxygen plasma to increase the hydrophilicity. It has been shown that treatment with oxygen plasma results in very low water contact angles that are stable over long times. ${ }^{7}$ After spin-coating the films underwent no further treatment but were investigated as-spun. However, we exclude the possibility that the increased values of $\mathrm{R}_{\mathrm{gcl}}$ are a result of a non-equilibrium conformation induced by the spin-coating process due to the following consideration: From spin-coating we would expect an elongation of the polymer rings in direction of the flow, i.e. in radial direction centered on the spinning axis. The geometry of the GISANS experiment leads to a beam footprint that covers only a narrow area along the beam direction across the sample center. Within this area the polymer rings would be elongated mainly along the beam direction and probably even narrowed perpendicular to it. Due to the nature of the GISANS experiment the dimension of the scattering objects perpendicular to the beam is determined. Therefore, a non-equilibrium conformation after the spin-coating would rather lead to reduced values of $\mathrm{R}_{\mathrm{gc}}$ and as a consequence, we can rule out such an effect.

Small angle X-ray scattering (SAXS) investigations were carried out on the dilute cyclic PNIPAM solution using a Ganesha 300 XL SAXS-WAXS instrument from SAXSLAB ApS, Copenhagen, Denmark. A Cu anode $\left(\mathrm{K}_{\alpha}\right)$ at a wavelength of $\lambda=0.1542 \mathrm{~nm}$ was used as the X-ray source. The detector was a 2D Pilatus 300K detector. About 50 microliter sample was filled in a quartz cuvette. During temperature-dependent measurements, the sample's temperature was stabilized for 15 min before data collection using a circulating bath thermostat from Julabo, Germany. The sample-detector-distance amounts to $401 \mathrm{~mm}$, all measurements were performed in a fully evacuated chamber. Absolute scattering intensities from the cyclic PNIPAM rings were obtained by standard data reduction procedures, accounting for the scattering of water and the empty cell. The 2D SAXS images were azimuthally averaged.

X-ray reflectivity curves were recorded for the three films on a Bruker D8 Advance in $\theta / 2 \theta$ geometry at an X-ray wavelength of $1.54 \AA$. The thicknesses of the three samples were determined to be $117 \mathrm{~nm}, 9.8 \mathrm{~nm}$, and $3.3 \mathrm{~nm}$.

The GISANS investigations were conducted at the high-flux SANS instrument D22 at the Institut Laue-Langevin (ILL) in Grenoble, France. A neutron beam with a wavelength of $6 \AA$ (size: $10 \times 1 \mathrm{~mm}^{2}$ ) was utilized. It impinged on the samples under an incident angle of $0.59^{\circ}$. As it lied above the critical angle of total external reflection of the polymers and the silicon, the full depth of the PNIPAM films was probed. As a consequence, an ensemble average of the structure of all the chains was carried out. The collimation was set to $17.6 \mathrm{~m}$ and the sample-to-detector distance to $8 \mathrm{~m}$. A two-dimensional ${ }^{3} \mathrm{He}$ detector with $128 \times 128$ pixels of $7.5 \times 7.5 \mathrm{~mm}^{2}$ each was employed. The reflection geometry was needed for the study of the thin films, as the scattering volume is considerably larger as for the transmission geometry. Long acquisition times above $8 \mathrm{~h}$ per sample were needed for this GISANS study. As we focused on the confinement effects arising for small PNIPAM rings, the scattering intensity from each individual chain was particularly low. 

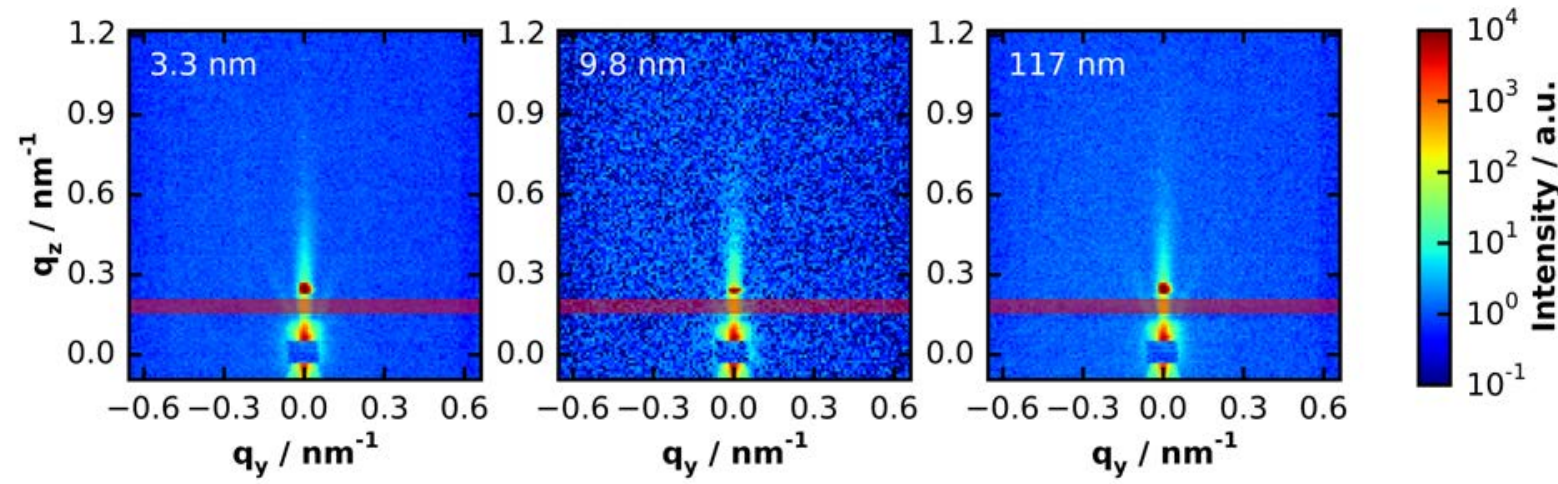

Figure S2. 2D GISANS scattering patterns of the three investigated samples. The film thickness is indicated in the upper right corner. The intensity is logarithmically color coded as depicted on the right. The data evaluation focusses on line cuts indicated by the red shaded area.

Figure S2 shows the 2D GISANS scattering patterns obtained from the experiment. The direct beam is shielded by an absorber, the specular peak is clearly visible at $\mathrm{q}_{\mathrm{z}}=0.25 \mathrm{~nm}^{-1}$. The data evaluation focuses on a horizontal line cut through the 2D GISANS data at the position of the Yoneda peak of PNIPAM. Five lines of the detector (corresponding to $\Delta \mathrm{q}_{\mathrm{z}}=0.05 \mathrm{~nm}^{-1}$ ) were summed up for improved statistics, indicated by the red shaded area in Figure S2. Due to a radial invariance around the sample normal, the scattering data were symmetric with respect to the vertical center. Thus, statistics were further improved by using both sides of the horizontal line cut for the analysis.
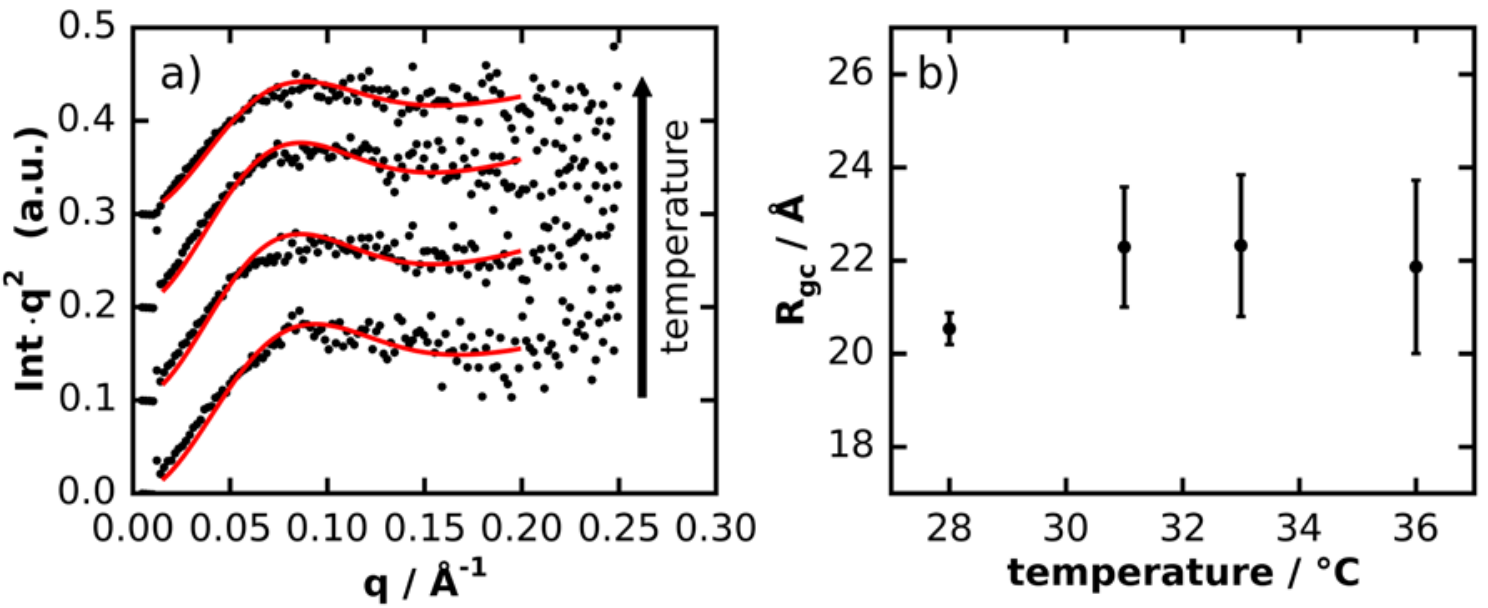

Figure S3. a) Kratky plot of the azimuthally averaged SAXS data. Black dots are data; red lines correspond to the fits described in the text. Curves are shifted vertically for clarity. The temperature increases from bottom to top. b) Obtained radii of gyration from the fits in a) for the different temperatures.

Fitting of the SAXS data was achieved analogous to the fits of the GISANS measurements. The azimuthally averaged data was normalized in a Kratky representation and fitted by the same function, a Padé approximant of equation (2). ${ }^{8}$ The data and the fits are depicted in Figure S3a for the different investigated temperatures. A summary of the obtained unperturbed radius of gyration in dependence of the temperature is shown in Figure S3b. The radii of gyration obtained for the solutions with $31^{\circ} \mathrm{C}, 33^{\circ} \mathrm{C}$ and $36^{\circ} \mathrm{C}$ are very close together at $2.2 \mathrm{~nm}$. In the solution at 28 ${ }^{\circ} \mathrm{C}$ a radius of gyration slightly lower $(20.5 \mathrm{~nm})$ was obtained from the fit. The discussion within the main text is lead with a radius of gyration of $2.2 \mathrm{~nm}$.

\section{References}

1. Qiu, X.-P.; Tanaka, F.; Winnik, F. M. Macromolecules 2007, 40, 7069-7071.

2. Satokawa, Y.; Shikata, T.; Tanaka, F.; Qiu, X.-P.; Winnik, F. M. Macromolecules 2009, 42, $1400-1403$. 
3. Schubert, D. W.; Dunkel, T. Mater. Res. Innovations 2003, 7, 314-321.

4. Müller-Buschbaum, P. J. Phys.: Condens. Matter 2003, 15, R1549.

5. Kaplan, W.; Chatain, D.; Wynblatt, P.; Carter, W. C. J. Mater. Sci. 2013, 48, 5681-5717.

6. Müller-Buschbaum, P. Eur. Phys. J. E 2003, 12, 443-448.

7. Shang, I. C.; Jenq-Gong, D. Jpn. J. Appl. Phys. 2014, 53, 11 RA01.

8. Rubio, A. M.; Alvarez, G.; Freire, J. J. Polymer 2008, 49, 628-634. 